\title{
Selected papers from the Tenth Chinese National Conference on X-Ray Diffraction and ICDD Workshop
}

The Tenth Chinese National Conference on X-Ray Diffraction and ICDD Workshop was held in Shanghai, China from Oct. 12 to 15,2009 . The conference and workshop were jointly organized by the Commission on Powder Diffraction, Chinese Society of Crystallography, the Commission on X-ray Diffraction, Chinese Society of Physics, the National Natural Science Foundation of China, the International Centre for Diffraction Data (ICDD), and the Beijing Ceramic Society. The local organizers were from the Shanghai Jiaotong University, and the Shanghai Institute of Ceramics of the Chinese Academy of Sciences. There were over 260 total participants from universities, institutions, and industry. The commercial vendors came from China, Europe, Japan, and the United States.

The first day's (Oct. 12) agenda comprised five invited plenary presentations and four talks from commercial vendors. The second day (Oct. 13) was solely devoted to an ICDD workshop in a single general session. The third day (Oct. 14) was occupied by 70 oral presentations in three parallel sessions with themes of new methods, theory, applications, and characterization of low-dimensional materials.

Prior to the conference, Dr. Ting C. Huang, Editor-inChief (EIC) of the Powder Diffraction (PD) journal, and I discussed the possibility of selecting some contributions from the participants to publish in a special issue of PD. The purpose is to introduce the research and applications of $\mathrm{X}$-ray diffraction done by the Chinese community to the researchers from other parts of the world. Dr. Ting C. Huang attended the conference and gave an introduction to both the history and the present status of PD. His talk generated many interests, and a large number of papers were submitted after the conference. After the standard peer-review process, nine of the conference papers along with other four regular technical articles submitted to PD were selected to be included in this special issue.

The papers were subjected to the same editorial process as that for regular technical articles of PD. They were first reviewed/edited by the Conference Organizing Committee and then were finally edited by the EIC of PD, Dr. Ting C. Huang. Huang must have spent numerous hours on editing these papers to ensure that they meet the requirements of PD, and we thank him for his efforts very much.

It should be pointed out that these selected papers represent some of the diffraction researches and applications recently conducted by Chinese scientists, and we hope that PD readers enjoy reading them.

In recent years, the number of diffractometers has been increased tremendously in China, beneficial from around $10 \%$ GDP annual rate in Chinese economy. Diffraction technique has become a routine tool for the characterization of materials in academic research and quality control in industry. Advances and breakthroughs in this research field can be anticipated. I hope that we have more opportunities in the future to introduce timely research works done in China to the $\mathrm{PD}$ readers.
Xiaolong Chen Editor 